\title{
Several Color Appearance Phenomena in Color Reproduction
}

\author{
Qin-ling Dai ${ }^{1, a}$, Xiao-zhou $\mathrm{Li}^{2^{\star}, \mathrm{b}}, \mathrm{Ai} \mathrm{Xu}^{2}$ \\ ${ }^{1}$ School of Materials Engineering (Southwest Forestry University), Kunming, China 650224 \\ ${ }^{2}$ Key Laboratory of Pulp \& Paper Science and Technology (Shandong Polytechnic University), \\ Ministry of Education, Ji'nan, China, 250353 \\ adaiqinling@126.com, bcorresponding author: lixiaozhou2000@163.com
}

\begin{abstract}
Keywords: color reproduction, color appearance, color appearance phenomena
\end{abstract}
\begin{abstract}
Color perceived performance was influenced by various color appearance phenomena caused by varying viewing conditions in color reproduction process. It is necessary to do some research on the color appearance phenomena to represent the color appearance models qualitatively and quantitatively and accurate color reproduction easily. Only the phenomena were studied thoroughly, could the color transmission and reproduction be well performed. The color appearance and common color appearance phenomena of color reproduction were analyzed in this paper. And the basic theory of color appearance in color reproduction was also studied.
\end{abstract}

\section{Introduction}

High fidelity digital printing plays an important role in high fidelity color transmission and reproduction and it is one of the most important techniques to perform high fidelity color reproduction. High fidelity digital printing helps to perform accurate color reproduction of the original which can't be performed because of paper and ink in traditional printing process [1]. In color printing, the color difference caused by paper, ink and viewing conditions is various. The difference is not only colorimetric difference but also different color appearance phenomena. While the different color appearance phenomenon is the leading factor to influence the color vision perceived. The reproduction quality is evaluated using the visual evaluation of perceived the relations among the colors including the single color reproduction when the reproduction is viewed under different viewing conditions. The purpose of high fidelity printing is not only to get the same colorimetric or color density but also to get the same color appearance. The common color appearance phenomena are studied in this paper according to the view conditions and the interaction of the colors. The results help to describe the color appearance models qualitatively and quantitatively and accurate color reproduction easily.

\section{Viewing Conditions}

How to perform the color information high fidelity reproduction is one of the important fields in color science all the time. The perception is different when the same color is viewed under different conditions. The viewing conditions include lighting condition, medium, background, surround and the observer [2]. And the relations between the viewing conditions can be shown as in Fig. 1.

In which, area 1 is color pixel stimulus, i.e., the object color sample. Usually, viewing field is $2^{\circ}$ area. Area 2 is the core area adjacent to area 1 , the object color sample. And area 2 is called as the neighborhood area of the color pixel stimulus. Area 3 is often set as the background area which stretches from the area 2 to the $10^{\circ}$ viewing angle area. When the color of the neighborhood area, $2^{\circ}$ view angle area, is same to the color of the background, area 3, the background area range can stretch from the boundary of the area 1. Area 4 is the surround area which usually is the area out of the background. Although the difference between the area 1 and the area 2 is tiny and is ignored in high fidelity color printing, the relation between area 1 and area 2 in prepress color image processing is paid more and more attention, especially in spatial color gamut mapping. The relation between the 
object color stimulus and the pixels of its neighborhood area is one of the important contents in the color image processing in prepress.

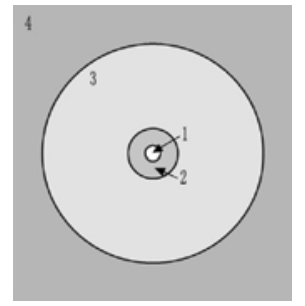

Fig.1 Viewing conditions and its correlations

\section{Color Appearance Phenomena}

Color appearance phenomenon is that the perceived color changes with the viewing conditions changing. Even under the same viewing conditions, the color appearance perceived is different. It is that the perceived color stimuli are influenced by the neighborhood pixels. The phenomenon that the stimulus is influenced by the spatial distribution of its neighborhood pixel stimulus is color appearance phenomenon [3]. So it is important and necessary to pay more attention to the research on color appearance phenomena.
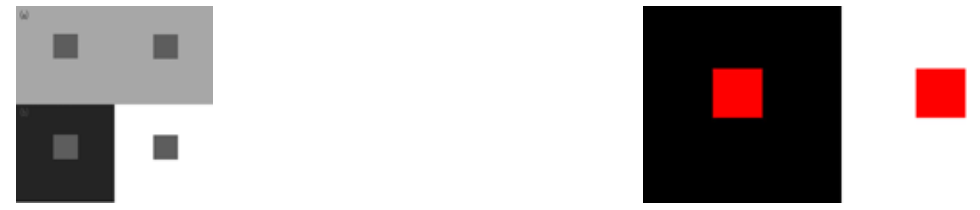

Fig.2 Simultaneous Contrast/ Induction Fig. 3 Influence of simultaneous contrast on color patch

Simultaneous contrast/ induction is one common color appearance phenomenon in prepress image processing and color displaying on monitor. It can be showed as in Fig. 2.

In which, the perception of the two color patches with the same gray value under the same gray background is consistent (sub-picture $a$ ). While the perception is inconsistent if the backgrounds are different. The color patch seems to lighter under the dark background and the other is darker under the white background (sub-picture $b$ ). The value of the gray background is $35 \%(a)$, and the values of background $\mathrm{b}$ in sub-picture $(b)$ are $100 \%$ for the dark and $0 \%$ for the white. The value of the object color patch is $64 \%$.

In Fig.3, the two color patches with the same chromatic color value ( $L: 55 a: 80, b: 70$ ), which lies at the background of dark ground is lighter and more brilliant than that lies at the background of white.

Two color patches with the same chromatic color value $(L: 88, a:-80, b: 80)$ is perceived greener under the red background than that under the white background (Fig. 3). And they are perceived with green under the yellow background while with yellow under the blue background (Fig. 5).

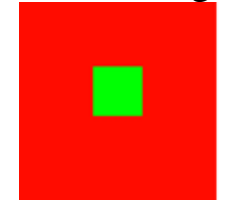

Fig. 4 Color Induction Phenomenon:

The background colors are red and white for the

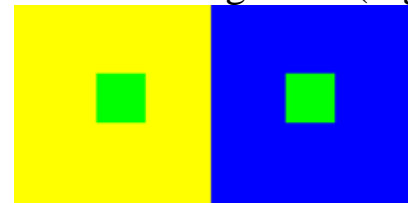

Fig. 5 Color Induction Phenomenon: The background colors are yellow and blue for the object color

Simultaneobjectoffltordst or color induction causes the color vision drift of perception which makes the whole color appearance change when the background of color stimulus is changed. These color vision drifts abide by the opponent color theory, i.e., it drifts along the complementary colors [4]. It shows that the color stimulus will be darker on the lighter background while be lighter on the lighter background. Red-green and yellow-blue will cause the corresponding induction color vision, i.e., red will induct the green, green will induct the red, yellow will induct the blue and blue will induct the yellow. 
Crispenining Phenomenon. The color difference between the color patches with similar color value under the same background is greater when the object color value is similar to that of the background. Otherwise, the difference is smaller when the object color value is obviously different from that of the background. It is shown in Fig. 6.

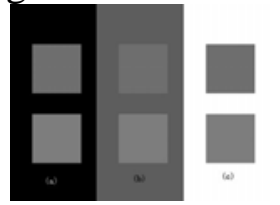

Fig. 6 Color difference crispening of achromatic color

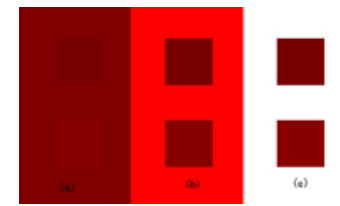

Fig. 7 Crispening of the chromatic color patches

A couple of gray color patches of crispening are shown in Fig. 6, in which the background color value is $(0,4,100)$, and the color patch 1 is 60 , color patch 2 is 65 . The lightness difference of the gray color patches under the gray background is greater than that under the white or dark background. So does the chromatic color stimuli (Fig. 7).

Fig. 7 shows the red color samples' crispening, in which the RGB value of the background is (R128, G0, B0), (R255, G0, B0) and (R255, G255, B255). Color patch 1 is $(R 120, G 0, B 0)$ and color patch 2 is $(R 136, G 0, B 0)$.

Color Difference Spreading. When the spatial distribution frequency of color samples increase or the size of color samples diminish, the simultaneous contrast will disappear and the spreading will emerge. The spreading effect is the mix of the color stimulus and its surround viewing condition apparently. Fig. 8 shows the simultaneous contrast effect and the spreading along the single color direction.

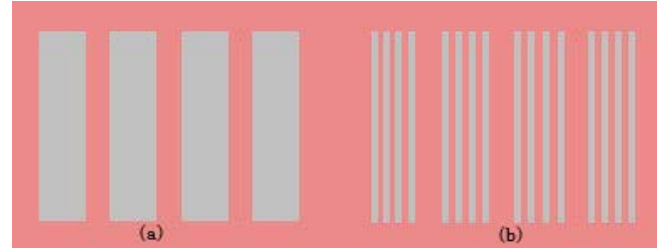

Fig. 8 Simultaneous contrast and Spreading effect

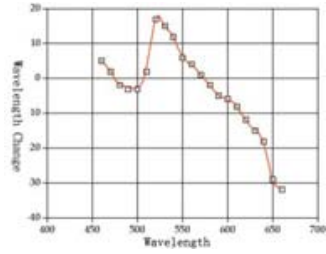

Fig.9 Bezold-Brucke Effect

It shows that the gray color patches with different frequency are shown on the same background of red. In which, the color value of red background is (R236, G138, B138) and the value of gray color patch is (R192, G192, B192). For the color patches with low frequency ( $a$ array), the perception of the color patches is slightly green while the spreading phenomenon occurs in the color patches with high frequency ( $b$ array), i.e., the perception is lightly pink for the gray ribbons mix with the red background. The simultaneous contrast changes to the spreading effect will be observed when the viewing distance is changed.

Bezold-Brucke color tone drift effect. The color tone perceived drifts with the lightness of spectra changes. The relation between the energy perceived by human eyes and the color vision is nonlinear information process. So the relation between color appearance perceived and the wavelength is dynamic for various monochromatic light under the changing viewing conditions. The phenomenon effect is showed in Fig. 9. In order to keep the consistent color tone, the wavelength changes ten times correspondingly the lightness of the change when the lightness changes [5].

Hunt Effect. Hunt effect is that colorfulness increases with luminance. It underlines the effect of absolute lightness on the color appearance. Hunt effect is a common phenomenon in cross-media color reproduction. However it is out of consideration in foundation colorimetry and should be predicted in color appearance model.

Stevens Effect. Stevens effect is that relative lightness increases with luminance of the adaptive field. It can be illustrated in Fig. 10. The dark tone will be darker while the light tone will be lighter with the luminance increases, i.e., the contrast increases.

Discounting-the-illuminant. Discounting-the-illuminant refers to the cognitive ability of observers to interpret the colors of the objects based on the illuminated environment in which they are viewed. This allows the observers to perceive colors of the objects independent of changes in the 
illumination and is consistent with the object. It is important and necessary to found the color appearance model for both reflector, e.g., paper, and spontaneous illuminant, e.g., monitor. For the color appearance of printing materials, the discount-the-illuminant should be considered while for the monitor it can be ignored.

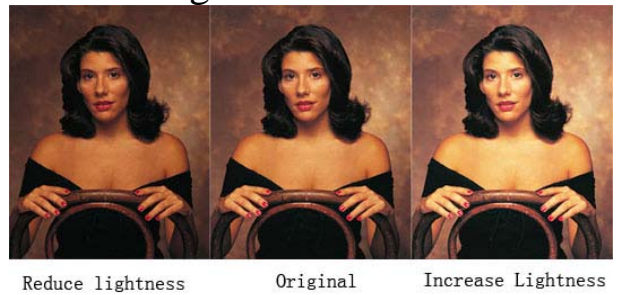

Fig. 10 Stevens Effect

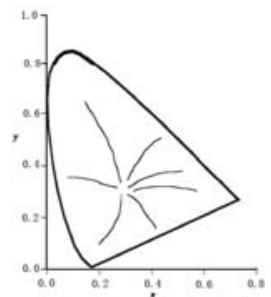

Fig. 11 The constant tone in CIE1931xyY

Abney Effect. Abney effect is that hue changes with colorimetric purity. The colorimetric purity of the monochromatic light will change when it mixes with the white light. The color tone will change for the monochromatic light with different lightness according to Bezold-Brucke color tune drift effect. The phenomenon can be illustrated as in Fig. 11. From the chart, we can see that the constant color tone is nonlinear.

Others. In color reproduction and perception, there are also other color appearance phenomena because of the changing viewing conditions, e.g., Helmholtz-Kohlrausch effect, Helson-Judd effect, Brtleson-Breneman effect, etc.

\section{Summary}

In this paper, we described some typical color appearance phenomena which have important influence on color reproduction and perception. In color reproduction, especially in the cross-medium reproduction of colors, it is necessary to do some research on the color appearance phenomena to represent the color appearance models qualitatively and quantitatively. Only the phenomena were studied thoroughly, could the color transmission and reproduction be well performed.

\section{Acknowledgements}

This work was financially supported by the Yunan Natural Science Foundation (Grant No. 2012FD029), China and Director Fund of Key Laboratory of Pulp \& Paper Science and Technology (Shandong Polytechnic University), Ministry of Education, Ji’nan, China( Grant No. 08031310).

\section{References}

[1] X.Z. Li: Study on Color Gamut Mapping Theory and Method Based on Color Appearance Model of High Fidelity Color Reproduction. South China University of Technology 2012.

[2] W.J. Hu, S.Q. Tang, F.Z. Zhu: Modern Color Science and Application (Beijing Institute of Technology Press, Beijing 2007).

[3] Mark D. F. : Color Appearance Models(John Wiley \& Sons Ltd, England, 2005).

[4] Steven K. Shevell. The Science of Color 2nd (Elsevier, UK 2003).

[5] N.F. Liao, J.S. Shi, W.M. Wu: An Introduction to Digital Color Management System (Beijing Institute of Technology Press, Beijing 2009). 